\title{
Combined genetic mutations and DNA- methylated genes as biomarkers for endometrial cancer detection from cervical scrapings
}

Phui-Ly Liew ${ }^{1,2}$, Rui-Lan Huang ${ }^{3,4,5}$, Tzu-I Wu ${ }^{5,6}$, Chi-Chun Liao, ${ }^{3,5}$, Chien-Wen Chen ${ }^{3,5}$, Po-Hsuan Su 4 , Hui-Chen Wang ${ }^{5}$, Yu-Chun Weng ${ }^{4}$ and Hung-Cheng Lai ${ }^{3,4,5,7,8,9^{*}}$ (D)

\begin{abstract}
Background: Endometrial cancer is a common gynecologic cancer. Noninvasive molecular biomarkers for triage of high-risk patients for invasive procedures are needed. Based on the success of cytological Pap smear screening, cervical scrapings are a good source of DNA for molecular testing. In addition to genetic lesions, DNA methylation is a promising biomarker. We assessed the usefulness of combining genetic and epigenetic biomarkers from cervical scrapings to detect endometrial carcinomas.
\end{abstract}

Methods: We performed a retrospective case-control study of 96 consecutive cervical scrapings from patients with abnormal uterine bleeding who underwent surgery for diagnostic evaluation. Thirty and 16 cases were diagnosed with type I and type II endometrial cancers, respectively. The remaining non-cancer cases included normal endometrium ( $n=12)$, benign uterine lesions $(n=20)$, and endometrial hyperplasia ( $n=18)$. Quantitative methylation-specific PCR and mass spectrometry were used for DNA methylation and genetic mutation analysis. Logistic regression was used to evaluate the clinical performance of these candidate biomarkers.

Results: We tested the effectiveness of the methylation status of four genes (BHLHE22, CDO1, TBX5, and HAND2) in endometrial cancer detection. The area under the receiver operating characteristic curves ranged from 0.703 to 0.878, and panels of hypermethylated BHLHE22/CDO1/HAND2 (87.0\% sensitivity and 86.0\% specificity) and BHLHE22/ CD01/TBX5 (89.1\% sensitivity and 80.0\% specificity) showed significant differences and could distinguish benign from malignant endometrial lesions. The sensitivity and specificity in endometrial cancer detection for BHLHE22/ CDO1 were $84.8 \%$ and $88.0 \%$, respectively. Both type I and II endometrial carcinomas could be detected using a BHLHE22/CDO1-based methylation profile, suggesting that they may have common epigenomes. Moreover, PTEN and TP53 mutations were found in 63.3\% of type I and $93.6 \%$ of type II endometrial cancers. Unexpectedly, PTEN and TP53 mutations were commonly found in cervical scrapings of the normal endometrium (25\% and 33.3\%, respectively) and in cases with benign uterine lesions (10\% and 50\%, respectively). Finally, combinations of any one mutation of PTEN and TP53 mutations had a sensitivity of $91.3 \%$, but a specificity of only $42.0 \%$.

Conclusions: Adding PTEN/TP53 mutation testing to BHLHE22/CDO1-based methylation testing did not improve the detection of endometrial cancer.

Keywords: Endometrial cancer detection, Cervical scrapings, Mutation, Methylation, Biomarkers

\footnotetext{
*Correspondence: hclai30656@gmail.com; hclai@s.tmu.edu.tw

${ }^{3}$ Department of Obstetrics and Gynecology, Shuang Ho Hospital, Taipei

Medical University, New Taipei, Taiwan

${ }^{4}$ Translational Epigenetic Center, Shuang Ho Hospital, Taipei Medical

University, New Taipei, Taiwan

Full list of author information is available at the end of the article
}

(c) The Author(s). 2019 Open Access This article is distributed under the terms of the Creative Commons Attribution 4.0 International License (http://creativecommons.org/licenses/by/4.0/), which permits unrestricted use, distribution, and reproduction in any medium, provided you give appropriate credit to the original author(s) and the source, provide a link to the Creative Commons license, and indicate if changes were made. The Creative Commons Public Domain Dedication waiver (http://creativecommons.org/publicdomain/zero/1.0/) applies to the data made available in this article, unless otherwise stated. 


\section{Background}

Endometrial cancer (EC) is the most common female genital tract malignancy in developed countries [1]. Although abnormal or dysfunctional uterine bleeding is the most frequent symptom of EC, only $10 \%$ of postmenopausal women with this symptom have EC. The most common diagnostic test for EC is transvaginal ultrasound, which measures the thickness of the endometrium. Unfortunately, transvaginal ultrasound cannot reliably distinguish between benign and malignant lesions [2]. Moreover, a cutoff value for an endometrial thickness that warrants further hysteroscopy remains under debate. Thus, invasive procedures to obtain endometrial tissues by fractional dilatation, curettage, and hysteroscopic biopsy remain necessary. However, the chance of diagnosing EC using current methods is low, even in symptomatic patients. Consequently, noninvasive molecular markers with acceptable accuracy for EC screening, at least in symptomatic women, are much needed.

The endometrium is a highly proliferative and cyclically regenerative tissue, which makes it vulnerable to genetic and epigenetic changes. While estrogen drives endometrial cell proliferation, progesterone inhibits it and causes cell differentiation. Conditions associated with a functional dominance of estrogen over progesterone increase the risk for both endometrial hyperplasia and EC. EC is broadly classified into two histotypes: type I, which consists of predominantly endometrioid adenocarcinomas, and type II, which incorporates serous-type, clear-cell, and poorly differentiated carcinomas. The proposed hyperplasia-to-carcinoma sequence for type I EC involves unopposed estrogen activity with subsequent mutations or alterations in pro-growth molecular pathways. Moreover, genomic data from The Cancer Genome Atlas defined four molecular subtypes of EC [3]. The four molecularly defined cancer subtypes are: DNA polymerase $\varepsilon$ exonuclease domain mutation, microsatellite-instable, microsatellite-stable with fewer copy-number alterations (CNAs), and microsatellite-stable (serous-like) with more CNAs. A categorization of ECs into one of these four subgroups could potentially provide individuals with prognostic and predictive information [4].

Unfortunately, the application of such genetic information in endometrial screening remains limited. Loss of function of the phosphatase and tensin homologue (PTEN) tumor suppressor heralds the beginning of multistep carcinogenesis, and its somatic mutation and/or deletion is the most common genetic change in endometrial endometrioid adenocarcinoma [5]. PTEN lesions are present in $83 \%$ of sporadic EC cases [6] and have been proposed to serve as diagnostic markers for endometrial precancerous lesions [7]. In addition to PTEN, approximately $25 \%$ of all ECs have been found to harbor mutations in the tumor suppressor TP53 [8]. Although both PTEN and TP53 drive carcinogenesis in many malignancies, the mechanistic role of both genes in endometrial carcinogenesis has not been fully elucidated [9].

A recent investigation revealed the feasibility of testing such genetic mutations in cervical scrapings ("Pap smears") for EC detection [10, 11]. In addition to genetic anomalies, epigenetic alternations are also involved in complex cancer development [12]. Aberrant DNA methylation-associated transcriptional silencing in tumor suppressor genes is commonly observed in human cancers [13]. Two predominantly global methylation patterns in cancer have been generally acknowledged: DNA hypermethylation of specific gene promoters, leading to gene silencing ("localized hypermethylation"), and loss of methylation within highly repeated DNA sequences, leading to unstable genomes and aberrant expression of oncogenes ("global hypomethylation"). Thus, understanding epigenetic regulation in EC progression may open new avenues for EC detection.

In light of the above, epigenetic lesions could potentially serve as early detection biomarkers for EC. For example, an epigenome-wide methylation analysis revealed that Heart and Neural Crest Derivatives Expressed 2 (HAND2) was one of the most commonly hypermethylated and silenced genes in EC [14]. Li and colleagues also demonstrated that HAND2 plays a major role in endometrial stromal-epithelial signaling [15], and that, in the presence of progesterone, endometrial epithelial cell proliferation inhibits HAND2 upregulation in endometrial stroma. Thus, HAND2 methylation is a common and crucial molecular alteration in EC that could hold clinical implications [16, 17]. The feasibility of HAND2 methylation testing in cervical scrapings and its value for EC detection remain undetermined. Our previous comprehensive methylomics approach identified in EC tissues a panel of highly methylated genes, including BHLHE22/CDO1/CELF4, that were detectable in cervical scrapings [18]. Such studies provide proof-of-concept for new means of EC screening using epigenetics analyses.

The combination of genetic and epigenetic markers from cervical scrapings for EC detection is logical and appealing. However, to our knowledge, no studies have tested such an approach. Therefore, the aim of the present study was to test whether the use of panels of combined epigenetic and genetic markers derived from cervical scrapings could improve EC detection.

\section{Methods}

\section{Clinical samples}

Female participants (age range, 30-80 years) were enrolled in our case-control retrospective study from November 2015 to September 2017. These women had cervical scrapings performed because of abnormal or 
dysfunctional uterine bleeding, followed by surgery at the Taipei Medical University-Shuang Ho Hospital, New Taipei City. This study was approved by the Institutional Review Board of the Taipei Medical University-Shuang Ho Hospital, in accordance with the Declaration of Helsinki 2000 (Protocol no. N201902024). Informed consent was obtained from all participants. Age, histologic type/grade of lesion or tumor, and International Federation of Gynecology and Obstetrics stage were extracted from the hospital records. Following collection, cervical scrapings were immediately placed in a RNAlater Stabilization Solution (Ambion, Thermo Fisher Scientific) and stored at $-80^{\circ} \mathrm{C}$ for future analysis.

\section{Methylation analysis of BHLHE22, CDO1, HAND2, and TBX5 genes}

Genomic DNA was extracted from the cervical scrapings using the QIAmp DNA Mini Kit (QIAGEN, Hilden, Germany), $800 \mathrm{ng}$ of which was modified with bisulfite using an EZ DNA Methylation Kit (D5008; Zymo Research, Irvine, CA, USA) according to the manufacturer's instructions, and then dissolved in $70 \mu \mathrm{L}$ nuclease-free water. PCR products and quantitative methylation-specific polymerase chain reaction (qMSPCR) were performed using a LightCycler 480 SYBR Green I Master (Roche, Penzberg, Germany). Reactions were carried out in $20 \mu \mathrm{L}$ containing $2 \mu \mathrm{L}$ bisulfiteconverted DNA, $250 \mathrm{nmol} / \mathrm{L}$ of each primer, and $10 \mu \mathrm{L}$ Master Mix using the following thermal profiles: $95^{\circ} \mathrm{C}$ for $5 \mathrm{~min}$ (initiation), 50 cycles of $95^{\circ} \mathrm{C}$ for $10 \mathrm{~s}, 60^{\circ} \mathrm{C}$ for $30 \mathrm{~s}$, and $72^{\circ} \mathrm{C}$ for $30 \mathrm{~s} \mathrm{(amplification),} \mathrm{and} \mathrm{a} \mathrm{final} \mathrm{exten-}$ sion step at $72{ }^{\circ} \mathrm{C}$ for $5 \mathrm{~min}$. All gene amplifications were conducted using duplicate specimens. To calculate a relative target amount, only the respective crossing point $(\mathrm{Cp})$ values of the target, the reference gene for each sample, and a calibrator need to be determined using LightCycler software. To normalize the input DNA in each methylation-independent assay, we used the amount of a non-CpG region of a type II collagen gene (COL2A1) as internal reference [19]. DNA methylation levels were estimated by the difference in crossing point $(\triangle \mathrm{Cp})$ values using the following formula: $\mathrm{Cp}$ of target $\mathrm{Cp}$ value of COL2A1. We considered and simplified the percentage of methylated reference for each result using the $\Delta \mathrm{Cp}$ values for detecting DNA methylation [20,21]. The analytical sensitivities of candidate genes are shown in Additional file 1: Figure S1. Linear regression analysis was used to assess assay linearity. The serial dilution of COL2A1 showed that the DNA temple was 118 copies; the mean $\mathrm{Cp}$ value of $C O L 2 A 1$ was 35.8 . Therefore, we defined $\mathrm{Cp}$ values of $C O L 2 A 1>36$ as not detected for each candidate gene in our samples. BHLHE22-, CDO1-, and TBX5-specific primers were designed using Oligo 7.0 Primer Analysis software (Molecular Biology
Insights, Inc.). We used the primer sequences of HAND2 described in a previous study [14].

\section{Somatic mutation detection and analysis of TP53 and PTEN genes}

To identify PTEN and TP53 exomic mutations, we sequenced all exons of those two genes using the Illumina HiSeq2500 high-throughput genome sequencer (Illumina, Inc., San Diego, CA, USA). Next, 40 ng of DNA from each individual was used to construct a DNA library of 43 target regions using the QIAseq targeted DNA system (QIAGEN). Briefly, DNA was enzymatically fragmented and end-repaired in a $25 \mu \mathrm{L}$ solution containing $2.5 \mu \mathrm{L} 10 \times$ fragmentation buffer and $5 \mu \mathrm{L}$ fragmentation enzyme mix. The reaction was carried out at $4{ }^{\circ} \mathrm{C}$ for $1 \mathrm{~min}, 32^{\circ} \mathrm{C}$ for $24 \mathrm{~min}$, and $65^{\circ} \mathrm{C}$ for $30 \mathrm{~min}$. Immediately after the reaction, $10 \mu \mathrm{L} 5 \times$ ligation buffer, $5 \mu \mathrm{L}$ DNA ligase, $2.8 \mu \mathrm{L}$ of $25 \mu \mathrm{M}$ bar-coded adapters, and water were added for a total volume of $50 \mu \mathrm{L}$. The reaction was then continued at $20^{\circ} \mathrm{C}$ for $15 \mathrm{~min}$. To ensure the complete removal of free barcoded adapters, each reaction was purified twice using a bead-system (QIAGEN). In a total volume of $20 \mu \mathrm{L}$, purified DNA was mixed with $10 \mathrm{nM}$ of each target primer, $400 \mathrm{nM}$ forward primer, $1 \times$ PCR buffer, and $0.8 \mu \mathrm{L}$ HotStarTaq DNA polymerase. The PCR enrichment conditions were: $95^{\circ} \mathrm{C}$ for $13 \mathrm{~min}, 98^{\circ} \mathrm{C}$ for $2 \mathrm{~min}$, six cycles of $98^{\circ} \mathrm{C}$ for $15 \mathrm{~s}, 65^{\circ} \mathrm{C}$ for $15 \mathrm{~min}$, and $72{ }^{\circ} \mathrm{C}$ for $5 \mathrm{~min}$. Each reaction mixture was purified to remove unused primers. The enriched DNA was combined with $400 \mathrm{nM}$ universal primer, $400 \mathrm{nM}$ index primer, $1 \times$ PCR buffer, and $1 \mu \mathrm{L}$ HotStarTaq DNA polymerase in a total volume of $20 \mu \mathrm{L}$. The universal PCR conditions were: $95^{\circ} \mathrm{C}$ for $13 \mathrm{~min}$, $98^{\circ} \mathrm{C}$ for $2 \mathrm{~min}$; 20 cycles of $98^{\circ} \mathrm{C}$ for $15 \mathrm{~s}, 60^{\circ} \mathrm{C}$ for 2 min, and $72^{\circ} \mathrm{C}$ for $5 \mathrm{~min}$. The DNA library was then purified and pooled for sequencing $(2 \times 100$ base pairs [BPs]). The raw output from each individual scraping was $>100 \mathrm{Mb}$, with an average target region depth $>30$, $000 \times$. The sequence of each read was trimmed based on its quality score (Q30), and a length $<45$ BPs of each read was discarded from the following analyses. The reads were aligned to the human hg19 reference genome using BWA-MEM (http://bio-bwa.sourceforge.net/), and variants were called using GATK Unified Genotyper (GATKLite version 2.3-9) [22]. After variant calling, Variant Effect Predictor (http://grch37.ensembl.org/ Homo_sapiens/Tools/VEP) was used to annotate the identified variants. We then selected the confidence of the mutations using alternate allele frequencies $\geq 0.3$ as a cutoff and removed synonymous substitutions (i.e., silent mutations in the encoded protein). All PTEN and TP53 mutations were displayed using MutationMapper software (https://www.cbioportal.org/mutation_mapper), as shown in Fig. 2a [23, 24]. The presence of a major 
mutation in each individual gene is shown as the maximum value of alternate allele frequencies in Fig. $2 \mathrm{~b}$.

\section{Statistical analysis}

Based on previous work, the area under the receiver operating characteristic curve (AUC-ROC) of hypermethylated HAND2 in the normal endometrium vs. EC tissues was 0.9 [14]. Consequently, we assumed that the AUCROC of hypermethylated HAND2 in cervical scrapings from normal and type I EC patients was 0.8. The null hypothesis AUC-ROC, type I error ( $\alpha$ ), and type II error $(\beta)$ values were $0.5,0.01$, and 0.05 , respectively, and the ratio of sample sizes of normal vs. type I EC tissue was 1.0, requiring at least 28 cases in both groups. We then added three samples to both groups to avoid a $10 \%$ failure rate in subsequent tests. Additionally, we simultaneously examined gene mutations and DNA methylation levels in DNA isolated from cervical scrapings from patients with endometrial hyperplasia $(n=18)$ and type II EC $(n=16)$. The Mann-Whitney nonparametric $U$ test and the Kruskal-Wallis test were used to identify significant differences in methylation levels between two categories and more than two categories, respectively. The associations between categorical clinical variables and methylation levels/genetic mutations were identified by the chi-square test for 2-by-2 categories. The combinations of methylated DNA levels were calculated using a logistic regression model, and each gene was weighted by a coefficient value. The cutoff values were evaluated by AUC-ROC analysis with the Youden method. A twotailed $P$ value $\leq 0.05$ was considered significant.

\section{Results}

Hypermethylation of BHLHE22, CDO1, HAND2, and TBX5 can be detected in cervical scrapings of patients with ECs Table 1 shows the clinical and pathological features of 96 patients from whom cervical scrapings and subsequent uterine tissue specimens were collected. These included scraped samples of normal endometrium $(n=$ $12)$, benign diseases $(n=20)$, endometrial hyperplasia $(n$ $=18)$, type I EC $(n=30)$, and type II EC $(n=16)$. The methylation levels of those four genes, in terms of $\Delta C p$ values, are displayed as dot plots in Fig. 1a. The lower the $\triangle \mathrm{Cp}$ value, the higher the gene's methylation status. BHLHE22, CDO1, and HAND2 methylation increased significantly from normal endometrium to endometrial hyperplasia, type I ECs, and type II ECs $(P<0.001)$ (Fig. 1a). Next, we calculated the AUC-ROCs for these four

Table 1 Demographics of clinical samples

\begin{tabular}{|c|c|c|c|c|c|}
\hline \multirow[t]{2}{*}{ Variables } & \multirow{2}{*}{$\begin{array}{l}\text { Normal } \\
\text { endometrium }\end{array}$} & \multirow{2}{*}{$\begin{array}{l}\text { Benign } \\
\text { diseases }\end{array}$} & \multirow[t]{2}{*}{ Hyperplasia } & \multicolumn{2}{|c|}{ Endometrial cancer (EC) } \\
\hline & & & & Type I & Type ॥ \\
\hline Number of cases & 12 & 20 & 18 & 30 & 16 \\
\hline Age (years) & $53.4 \pm 5.6$ & $46.2 \pm 5.8$ & $46.4 \pm 6.5$ & $55.3 \pm 6.9$ & $59.6 \pm 8.7$ \\
\hline \multicolumn{6}{|l|}{ Subtypes } \\
\hline Adenomyosis & & $1(5 \%)$ & & & \\
\hline Leiomyoma & & $10(50 \%)$ & & & \\
\hline Adenomyosis and leiomyoma & & $9(45 \%)$ & & & \\
\hline Endometrial hyperplasia & & & $12(42.9 \%)$ & & \\
\hline Atypical endometrial hyperplasia & & & $6(33.3 \%)$ & & \\
\hline \multicolumn{6}{|l|}{ Histotypes of cancer } \\
\hline Endometrioid & & & & $30(100 \%)$ & $3(18.8 \%)$ \\
\hline Serous & & & & 0 & $6(37.5 \%)$ \\
\hline Others & & & & 0 & $7(43.8 \%)$ \\
\hline \multicolumn{6}{|l|}{ FIGO stage of cancer } \\
\hline । & & & & $28(93.3 \%)$ & $10(62.5 \%)$ \\
\hline$\|$ & & & & 0 & $1(6.2 \%)$ \\
\hline III & & & & $1(3.3 \%)$ & $3(18.8 \%)$ \\
\hline IV & & & & $1(3.3 \%)$ & $2(12.5 \%)$ \\
\hline \multicolumn{6}{|l|}{ Histological grade of cancer } \\
\hline G1 & & & & $20(66.7 \%)$ & $4(25.0 \%)$ \\
\hline G2 & & & & $8(26.7 \%)$ & $2(12.5 \%)$ \\
\hline G3 & & & & 0 & $9(56.2 \%)$ \\
\hline Unknown & & & & $2(6.7 \%)$ & $1(6.2 \%)$ \\
\hline
\end{tabular}




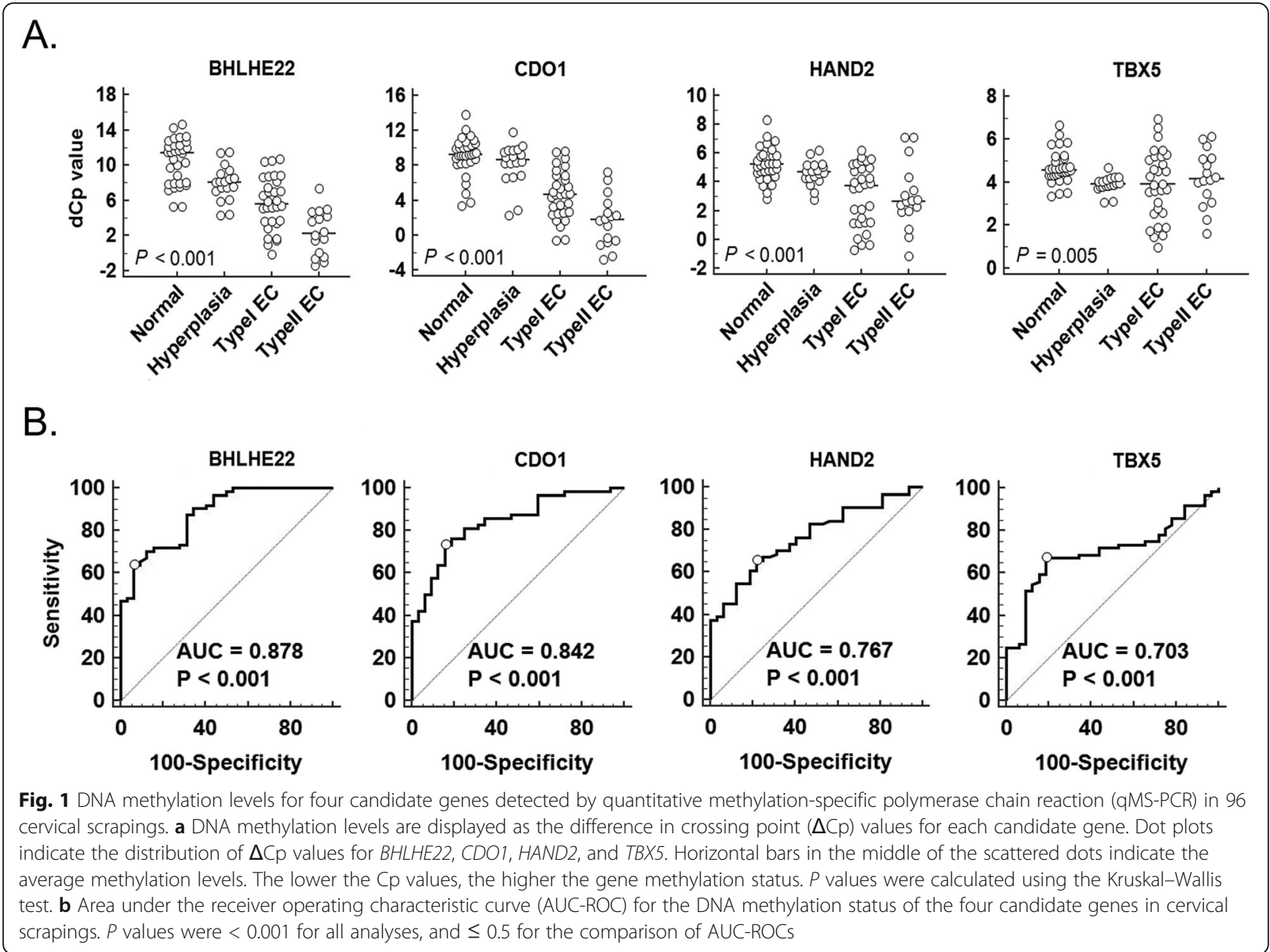

genes to determine the sensitivity and specificity of methylated DNA for cancer detection in cervical scrapings. The results ranged from 0.703 to 0.878 (Fig. 1b). BHLHE22 performed the best of all tested genes.

\section{PTEN and TP53 mutations are commonly detected in cervical scrapings from patients with a wide range of endometrial lesions}

Targeted sequencing of PTEN and TP53 revealed mutations across the whole exome of each gene in the control group (normal endometrium) and in the disease groups without hot spots (Fig. 2a). As expected, PTEN mutations were common in $63.3 \%$ of type I ECs, and TP53 mutations in $93.6 \%$ of type II ECs (Fig. 2b). TP53 mutations were also common in type I ECs (63.3\%), and these were primarily missense mutations. Unexpectedly, PTEN and TP53 mutations were commonly found in cervical scrapings of control patients and those with benign uterine lesions. PTEN and TP53 mutations could be detected in controls (25\% and $33.3 \%$, respectively) and patients with benign uterine lesions (10\% and $50 \%$, respectively) (Fig. 2b). Simultaneous PTEN and TP53 mutations correlated with disease progression from $8.3 \%$ (controls), to $10 \%$ (benign uterine lesions), to $34.7 \%$, in EC cancers.

Adding genetic mutations to epigenetic methylation aberrations compromises their performance as clinical biomarkers

For clinical applications, any disease-correlated genetic alterations were called positive, as shown in Fig. 3. For example, the sensitivities and specificities of PTEN and TP53 mutations for overall ECs were $52.2 \%$ and $86 \%$, and $71.1 \%$ and $62 \%$, respectively (Fig. $3 \mathrm{~b}$ and Table 2). In addition to those genomic assays, we assessed whether three combinations of DNA methylation levels of the BHLHE22, CDO1, TBX5, and HAND2 genes identified in our previous study [18] might provide the best performance for EC detection in terms of sensitivity and specificity. The sensitivities and specificities of combined DNA methylation were 87.0-89.1\% (BHLHE22/CDO1/ HAND2) and 80.0-86.0\% (BHLHE22/CDO1/TBX5), respectively. Adding genetic mutations increased the detection sensitivity of type I, but not type II ECs, but the specificity was severely compromised because of the 


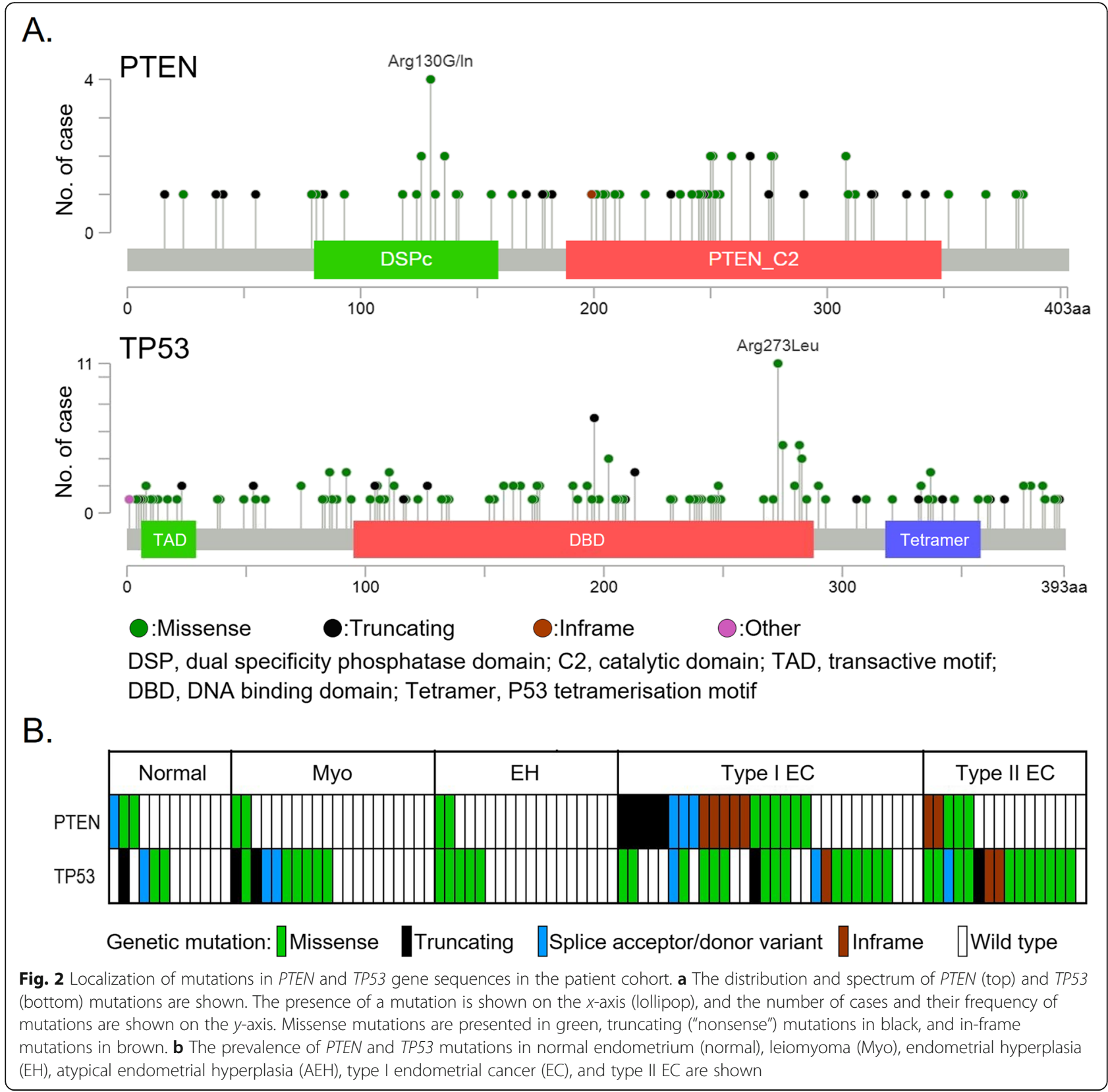

numerous mutations detected in patients without ECs (Fig. 3b).

\section{Discussion}

Endometrial cancer (EC) is the most commonly diagnosed gynecologic malignancy, and incidences and mortality rates are increasing at an alarming pace [1]. EC already has a high prevalence, and its morbidity and mortality rates continue to increase; however, to our knowledge, no existing screening method can effectively detect either precancerous lesions or early-stage cancer. The need for improved screening is particularly high because when detected early, EC survival rates dramatically improve. EC is a heterogeneous disease [25], and the risks of EC development are highly influenced by genetic and non-hereditary factors [26]. In light of the increase of high-risk populations due to the current aging and obesity epidemics, women with risk factors such as postmenopausal bleeding, breast cancer treatment with hormone therapy, and a family history of Lynch syndrome desperately need novel and noninvasive molecular biomarkers that can detect EC early. In addition to being useful for genetic mutation testing [11, 27], cervical scrapings could also play a functional role in methylation-based screening [28] to facilitate the detection of EC. To that end, this study was the first to 


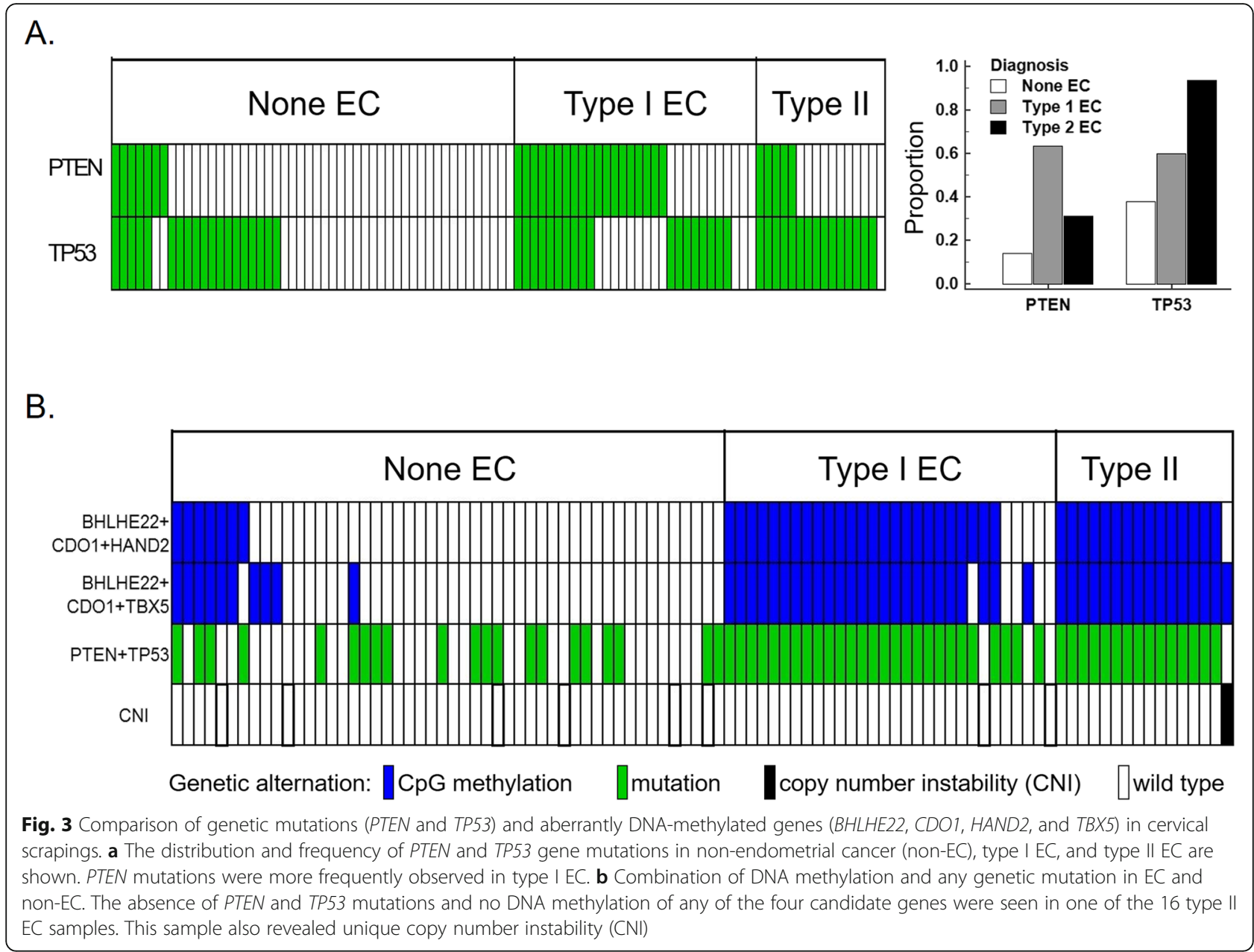

investigate whether combined testing of genetic mutations and epigenetic DNA methylation alterations from cervical scrapings would increase sensitivity and specificity for the detection of EC.

Our study provides supporting evidence that epigenetic biomarkers (i.e., a BHLHE22/CDO1-based panel) may be more useful than genetic mutation-based biomarkers for detecting EC. In the 96 cervical scrapings, we found high AUC-ROCs for four hypermethylated genes (BHLHE22, CDO1, HAND2, and TBX5, which were previously identified by our group [18]) in patients with type I and type II ECs compared with patients with normal endometrium and endometrial hyperplasia. These genes have low methylation levels in cervical lesions (Additional file 1: Table S1). We also demonstrated that three-gene panels of hypermethylated BHLHE22/ CDO1/HAND2 and BHLHE22/CDO1/TBX5 were significantly different between patients with and without EC. By contrast, PTEN and TP53 mutations were commonly found in cervical scrapings of healthy controls and those with benign gynecologic diseases. Clonal proliferations of nonmalignant cells and benign diseases have been described in the bone marrow and noncancerous tissue, and in endometriosis [29-32]. Although it these mutations might reflect benign or noncancerous endometrial lesions $[10,32]$, the mechanism underlying the mutational changes in normal endometrium and benign uterine lesions remains to be elucidated.

We also provide exploratory insights that ECs may share common epigenetic events, and that DNA methylation changes regardless of genetic heterogeneity and clinicopathology. ECs are conventionally classified into types I and II according to their clinicopathological characteristics and heterogeneity at the genetic level. In addition to these genetic events, the role of epigenetics combined with mutation-driven classification remains unknown. An important issue is whether different panels of DNA methylation profiles might be able to distinguish the two EC histotypes. Our previous study [18] identified BHLHE22, CDO1, and CELF4 methylation in cervical scrapings as excellent molecular biomarkers for EC detection. When detecting type II ECs, 14 samples, except for one of the serous type (92.9\%), showed hypermethylation of this three-gene panel. Our present study further 
Table 2 Performance of genetic mutations, and methylated gene combinations, in cervical scrapings

\begin{tabular}{|c|c|c|c|}
\hline Variables & Non-endometrial cancer & Endometrial cancer & $P$ value $^{a}$ \\
\hline Number of cases & 50 & 46 & \\
\hline \multicolumn{4}{|l|}{ Genetic mutation } \\
\hline PTEN & & & $<0.001$ \\
\hline Mutation & $7(14.0 \%)$ & $24(52.2 \%)$ & \\
\hline Wild type & $43(86.0 \%)$ & $22(47.8 \%)$ & \\
\hline TP53 & & & $<0.001$ \\
\hline Mutation & 19 (38.0\%) & $33(71.7 \%)$ & \\
\hline Wild type & $31(62.0 \%)$ & $13(28.3 \%)$ & \\
\hline Either mutation & & & $<0.001$ \\
\hline Mutation & $29(58.0 \%)$ & $42(91.3 \%)$ & \\
\hline Wild type & $21(42.0 \%)$ & $4(8.7 \%)$ & \\
\hline \multicolumn{4}{|l|}{ DNA methylation } \\
\hline $\begin{array}{l}\text { BHLHE22+CDO1 } \\
\text { (cutoff value }>-0.2)^{b}\end{array}$ & & & $<0.001$ \\
\hline High & $6(12.0 \%)$ & 39 (84.8\%) & \\
\hline Low & $44(88.0 \%)$ & $7(15.2 \%)$ & \\
\hline BHLHE22+CDO1+HAND2 (cutoff value $>0.22)^{b}$ & & & $<0.001$ \\
\hline High & $7(14.0 \%)$ & $40(87.0 \%)$ & \\
\hline Low & $43(86.0 \%)$ & $6(13.0 \%)$ & \\
\hline BHLHE22+CDO1+TBX5 (cutoff value $>-0.04)^{b}$ & & & $<0.001$ \\
\hline High & $10(20.0 \%)$ & $41(89.1 \%)$ & \\
\hline Low & $40(80.0 \%)$ & $5(10.9 \%)$ & \\
\hline
\end{tabular}

${ }^{a} P$ values were calculated by the chi-square test

${ }^{\mathrm{b}}$ Cutoff values were calculated by the maximum of Youden's distance of receiver operating characteristic curve

confirmed that both type I and II ECs can be detected by a BHLHE22/CDO1-based methylation profile. BHLHE22 (also known as BHLHB5) encodes a basic helix-loop-helix transcription factor and has been implicated in neural development, including cell growth, cell differentiation, and cell migration [33]. CDO1 is a tumor suppressor gene, and methylation of its promoter region has been found in numerous cancers [34, 35]. Additionally, T-box transcription factor 5 (TBX5) is a member of a phylogenetically conserved family of genes involved in the regulation of developmental processes. The function of TBX5 in cancer development is largely unclear [36-38]. HAND2 is a progesterone-regulated stem cell polycomb group target gene that encodes a transcription factor expressed in the endometrial stroma to suppress estrogen-mediated signals. One previous study [14] reported that DNA methylation of HAND2 could be a key step in EC development and, thus, could potentially be used as a biomarker for the early detection of ECs.

Today, mutations in PTEN and TP53 are best documented in genetic lesions occurring in sporadic ECs. In our present and previous studies, PTEN mutations were detected in $63.3 \%$ of type I ECs and $31.2 \%$ of type II ECs
[5, 39]. Up to $93.6 \%$ of type II and $63.3 \%$ of type I EC patients harbored detectable TP53 mutations in their cervical scraping samples. Therefore, TP53 mutations are not restricted to type II endometrial serous carcinomas; they are also present in a subset of type I endometrial endometrioid carcinomas. The potential relationship between mutations in TP53 and PTEN, as observed in endometrial carcinoma, is still poorly known [40-42]. Furthermore, to ensure that genetic screening profiles are more informative, we assert that the mutation-based detection thresholds should be stricter, and that the criteria to determine cutoff points should be more specific. PTEN mutations seem to be of little importance in uterine cervical lesions [43]. Our supplementary data showed that PTEN and TP53 mutations had been found in 13\% and $9 \%$ of cervical squamous cell carcinoma cases, respectively (Additional file 1: Figure S2). Recently, Salk et al. reported that subclonal mutations in cancer evolutionary processes were ubiquitous and part of normal human aging. Therefore, great care must be taken to distinguish tumor-derived from age-associated mutations in high-sensitivity clinical cancer diagnostics [44].

Despite its notable findings, our study had several limitations. First, it was retrospective rather than prospective. 
Moreover, the samples examined were derived from patients with abnormal uterine bleeding. In a screening setting, endometrial premalignant lesions and cancers will include earlier stage cancers and asymptomatic women. Early detection and treatment prediction require further validation, both in prospective and unbiased cohorts. Additionally, we need to explore the value of combined genetic and epigenetic analyses of DNA obtained from cervical scrapings during a routine Pap test in asymptomatic women to detect hidden ECs. Second, cutoff values for candidate biomarker genes for research purposes might not be directly applicable to clinical settings or the wider population, thereby warranting further validation in larger, population-based studies. Third, we found mutation and methylation changes in cervical scrapings from women with normal endometrium and benign uterine diseases. These "background" readings could interfere with the sensitivity and specificity rates of the detection of ECs, necessitating some type of filtering. Finally, the highest methylation levels we detected were in fully cancerous tissues, atypical endometrial hyperplasia, or stage I diseases. Thus, future studies should concentrate on identifying biomarkers specific for these types of cells for early detection.

\section{Conclusions}

In conclusion, we demonstrated promising epigenetic biomarkers in cervical scrapings for EC screening to triage women with abnormal uterine bleeding for invasive procedures. These epigenetic biomarkers could broaden the scope of Pap testing and potentially be employed to detect ECs in the early stage, when the disease is easiest to treat. The value of adding genetic- to epigenetic-based biomarkers to detect $\mathrm{EC}$ requires further investigation.

\section{Supplementary information}

Supplementary information accompanies this paper at https://doi.org/10. 1186/s13148-019-0765-3

Additional file 1: Table S1. Performance of methylated gene combinations to detect endometrial cancer in scrapings of cervical lesions. Figure S1. The analytical sensitivity of target genes and limit of COL2A1 detection. Linear regression analysis was performed to assess assay linearity. ( $\mathbf{A}$ and $\mathbf{B}$ ) The $\Delta C p$ values and serial percentages of methylated DNA showed a high correlation $\left(R^{2}=0.998\right.$ and 0.99 for BHLHEE22 and CDO1, respectively). The mean $\triangle C p$ values of $0.1 \%$ methylated DNA were 7.87 and 7.45 for BHLHEE22 and CDO1, respectively. Additionally, when the DNA temple was 118 copies, the mean C $p$ value of COL2A1 was 35.8 (C). Therefore, we defined clinical samples with $C p$ values of $C O L 2 A 1>36$ as not detectable. Figure S2. PTEN and TP53 mutations in cervical squamous cell carcinoma. We visualized these two genetic mutations from cBioPortal (https://www. cbioportal.org/). Copy number alteration data including 278 samples from The Cancer Genome Atlas and the PanCancer Atlas studies were chosen for mutation analysis

\section{Abbreviations}

AUC: Area under a receiver-operating characteristic curve; $\mathrm{Cl}$ : Confidence interval; CNA: Copy number abnormality; CNI: Copy number instability; Cp: Crossing point; EC: Endometrial cancer; EH: Endometrial hyperplasia;
HAND2: Heart and neural crest derivatives expressed 2; Pap: Papanicolaou; PTEN: Phosphatase and tensin homologue; qMS-PCR: Quantitative methylation-specific polymerase chain reaction; ROC: Receiver operating characteristic; TCGA: The Cancer Genome Atlas

\section{Acknowledgements}

This work was financially supported of the Higher Education Sprout Project by the Ministry of Education (MOE) in Taiwan (DP2-108-21121-01-0-04-01, DP2-108-21121-01-0-04-02 and DP2-108-21121-01-0-04-03). This study was also supported by research grants from the Ministry of Science and Technology of Taiwan (MOST 108-2314-B-038-096) and Shuang Ho Hospital-Taipei Medical University (106TMU-SHH-14 and 107TMU-SHH-05).

\section{Availability of data and material}

The datasets used and/or analyzed during the current study are available from the corresponding author on reasonable request.

\section{Authors' contributions}

$\mathrm{HCL}$ designed the study and outlined the main conceptual ideas. RLH generated the primary datasets, and PHS, HCW, and YCW helped guide data generation and results interpretation. TIW, CCL, and CWC collected and contributed to the clinical samples and data analysis. PLL, RLH, and $\mathrm{HCL}$ wrote the article, and all authors provided critical feedback and helped shape the research, analysis, and manuscript. All authors read and approved the final manuscript.

\section{Ethics approval and consent to participate}

Ethical approval was granted by the Institutional Review Board of Taipei Medical University-Shuang Ho Hospital, in accordance with the Declaration of Helsinki, 2000 (Protocol no. N201902024). Informed consent was obtained from all participants in this study, who were de-identified.

Consent for publication

All authors have read and approved the manuscript for publication.

\section{Competing interests}

The authors declare that they have no competing interests.

\section{Author details}

${ }^{1}$ Department of Pathology, Shuang Ho Hospital, Taipei Medical University, New Taipei, Taiwan. ${ }^{2}$ Department of Pathology, School of Medicine, College of Medicine, Taipei Medical University, Taipei, Taiwan. ${ }^{3}$ Department of Obstetrics and Gynecology, Shuang Ho Hospital, Taipei Medical University, New Taipei, Taiwan. ${ }^{\top}$ Translational Epigenetic Center, Shuang Ho Hospital, Taipei Medical University, New Taipei, Taiwan. ${ }^{5}$ Department of Obstetrics and Gynecology, School of Medicine, College of Medicine, Taipei Medical University, Taipei, Taiwan. ${ }^{6}$ Department of Obstetrics and Gynecology, Wan Fang Hospital, Taipei Medical University, Taipei, Taiwan. ${ }^{7}$ Department of Clinical Pharmacology, Xiangya Hospital, Central South University, Changsha 410008, People's Republic of China. ${ }^{8}$ Institute of Clinical Pharmacology, Central South University; Hunan Key Laboratory of Pharmacogenetics, Changsha 410078, People's Republic of China. ${ }^{9}$ New Taipei, Taiwan.

Received: 22 April 2019 Accepted: 17 October 2019

Published online: 28 November 2019

\section{References}

1. Siegel RL, Miller KD, Jemal A. Cancer statistics, 2019. CA Cancer J Clin. 2019; 69(1):7-34.

2. Jacobs I, Gentry-Maharaj A, Burnell M, Manchanda R, Singh N, Sharma A, Ryan A, Seif MW, Amso NN, Turner G, et al. Sensitivity of transvaginal ultrasound screening for endometrial cancer in postmenopausal women: a case-control study within the UKCTOCS cohort. Lancet Oncol. 2011;12(1):38-48.

3. Cancer Genome Atlas Research N, Kandoth C, Schultz N, Cherniack AD, Akbani R, Liu Y, Shen H, Robertson AG, Pashtan I, Shen R, et al. Integrated genomic characterization of endometrial carcinoma. Nature. 2013;497(7447):67-73.

4. Kuhn E, Wu RC, Guan B, Wu G, Zhang J, Wang Y, Song L, Yuan X, Wei L, Roden $\mathrm{RB}$, et al. Identification of molecular pathway aberrations in uterine serous carcinoma by genome-wide analyses. J Natl Cancer Inst. 2012; 104(19):1503-13. 
5. Orbo A, Kaino T, Arnes M, Kopp M, Eklo K. Genetic derangements in the tumor suppressor gene PTEN in endometrial precancers as prognostic markers for cancer development: a population-based study from northern Norway with long-term follow-up. Gynecol Oncol. 2004;95(1):82-8.

6. Mutter GL, Monte NM, Neuberg D, Ferenczy A, Eng C. Emergence, involution, and progression to carcinoma of mutant clones in normal endometrial tissues. Cancer Res. 2014;74(10):2796-802.

7. Pavlakis K, Messini I, Vrekoussis T, Panoskaltsis T, Chrissanthakis D, Yiannou P, Stathopoulos EN. PTEN-loss and nuclear atypia of EIN in endometrial biopsies can predict the existence of a concurrent endometrial carcinoma. Gynecol Oncol. 2010;119(3):516-9.

8. Lee EJ, Kim TJ, Kim DS, Choi CH, Lee JW, Lee JH, Bae DS, Kim BG. p53 alteration independently predicts poor outcomes in patients with endometrial cancer: a clinicopathologic study of 131 cases and literature review. Gynecol Oncol. 2010;116(3):533-8.

9. Abrao F, Modotti WP, Spadoto-Dias D, Bueloni-Dias FN, Leite NJ, Peres GF, Elias LV, Domingues MAC, Dias R. Concomitant p53 and PTEN immunoexpression to predict the risk of malignancy in endometrial polyps. Medicine (Baltimore). 2018;97(38):e12304.

10. Nair N, Camacho-Vanegas O, Rykunov D, Dashkoff M, Camacho SC, Schumacher CA, Irish JC, Harkins TT, Freeman E, Garcia I, et al. Genomic analysis of uterine lavage fluid detects early endometrial cancers and reveals a prevalent landscape of driver mutations in women without histopathologic evidence of cancer: a prospective cross-sectional study. PLoS Med. 2016;13(12):e1002206.

11. Wang Y, Li L, Douville C, Cohen JD, Yen TT, Kinde I, Sundfelt K, Kjær SK, Hruban $\mathrm{RH}$, Shih IM, et al. Evaluation of liquid from the Papanicolaou test and other liquid biopsies for the detection of endometrial and ovarian cancers. Sci Transl Med. 2018:eaap8793.

12. Koukoura O, Spandidos DA, Daponte A, Sifakis S. DNA methylation profiles in ovarian cancer: implication in diagnosis and therapy (Review). Mol Med Rep. 2014;10(1):3-9.

13. Schultz MD, He Y, Whitaker JW, Hariharan M, Mukamel EA, Leung D, Rajagopal $\mathrm{N}$, Nery JR, Urich MA, Chen $\mathrm{H}$, et al. Human body epigenome maps reveal noncanonical DNA methylation variation. Nature. 2015;523(7559):212-6.

14. Jones A, Teschendorff AE, Li Q, Hayward JD, Kannan A, Mould T, West J, Zikan M, Cibula D, Fiegl H, et al. Role of DNA methylation and epigenetic silencing of HAND2 in endometrial cancer development. PLoS Med. 2013;10(11):e1001551.

15. Li Q, Kannan A, DeMayo FJ, Lydon JP, Cooke PS, Yamagishi H, Srivastava D, Bagchi MK, Bagchi IC. The antiproliferative action of progesterone in uterine epithelium is mediated by Hand2. Science. 2011;331(6019):912-6.

16. Yang X, Wang CC, Lee WYW, Trovik J, Chung TKH, Kwong J. Long non-coding RNA HAND2-AS1 inhibits invasion and metastasis in endometrioid endometrial carcinoma through inactivating neuromedin U. Cancer Lett. 2018;413:23-34.

17. Buell-Gutbrod R, Cavallo A, Lee N, Montag A, Gwin K. Heart and Neural Crest Derivatives Expressed Transcript 2 (HAND2): a novel biomarker for the identification of atypical hyperplasia and Type I endometrial carcinoma. Int J Gynecol Pathol. 2015;34(1):65-73.

18. Huang RL, Su PH, Liao YP, Wu TI, Hsu YT, Lin WY, Wang HC, Weng YC, Ou YC, Huang TH, Lai HC. Integrated epigenomics analysis reveals a dna methylation panel for endometrial cancer detection using cervical scrapings. Clin Cancer Res. 2017;23(1):263-72.

19. Huang RL, Chang CC, Su PH, Chen YC, Liao YP, Wang HC, Yo YT, Chao TK, Huang HC, Lin CY, et al. Methylomic analysis identifies frequent DNA methylation of zinc finger protein 582 (ZNF582) in cervical neoplasms. PLoS One. 2012;7(7):e41060.

20. Ogino S, Kawasaki T, Brahmandam M, Cantor M, Kirkner GJ, Spiegelman D, Makrigiorgos GM, Weisenberger DJ, Laird PW, Loda M, Fuchs CS. Precision and performance characteristics of bisulfite conversion and real-time PCR (MethyLight) for quantitative DNA methylation analysis. J Mol Diagn. 2006;8(2):209-17.

21. Eads CA, Danenberg KD, Kawakami K, Saltz LB, Blake C, Shibata D, Danenberg PV, Laird PW. MethyLight: a high-throughput assay to measure DNA methylation. Nucleic Acids Res. 2000;28(8):E32.

22. Aligning sequence reads, clone sequences and assembly contigs with BWAMEM. [https://arxiv.org/abs/1303.3997v2]

23. Gao J, Aksoy BA, Dogrusoz U, Dresdner G, Gross B, Sumer SO, Sun Y, Jacobsen A, Sinha R, Larsson E, et al: Integrative analysis of complex cancer genomics and clinical profiles using the cBioPortal. Sci Signal 2013;6(269):pl1.

24. Cerami E, Gao J, Dogrusoz U, Gross BE, Sumer SO, Aksoy BA, Jacobsen A, Byrne CJ, Heuer ML, Larsson E, et al. The cBio cancer genomics portal: an open platform for exploring multidimensional cancer genomics data. Cancer Discov. 2012;2(5):401-4.
25. McConechy MK, Ding J, Cheang MC, Wiegand K, Senz J, Tone A, Yang W, Prentice L, Tse K, Zeng T, et al. Use of mutation profiles to refine the classification of endometrial carcinomas. J Pathol. 2012;228(1):20-30.

26. Lichtenstein P, Holm NV, Verkasalo PK, Iliadou A, Kaprio J, Koskenvuo M, Pukkala E, Skytthe A, Hemminki K. Environmental and heritable factors in the causation of cancer--analyses of cohorts of twins from Sweden, Denmark, and Finland. N Engl J Med. 2000;343(2):78-85.

27. Maritschnegg E, Wang $Y$, Pecha N, Horvat R, Van Nieuwenhuysen E, Vergote I, Heitz F, Sehouli J, Kinde I, Diaz LA Jr, et al. Lavage of the uterine cavity for molecular detection of Mullerian duct carcinomas: a proof-of-concept study. J Clin Oncol. 2015;33(36):4293-300.

28. Wentzensen N, Bakkum-Gamez JN, Killian JK, Sampson J, Guido R, Glass A, Adams L, Luhn P, Brinton LA, Rush B, et al. Discovery and validation of methylation markers for endometrial cancer. Int J Cancer. 2014;135(8):1860-8.

29. Steensma DP, Bejar R, Jaiswal S, Lindsley RC, Sekeres MA, Hasserjian RP, Ebert BL. Clonal hematopoiesis of indeterminate potential and its distinction from myelodysplastic syndromes. Blood. 2015;126(1):9-16.

30. Krimmel JD, Schmitt MW, Harrell MI, Agnew KJ, Kennedy SR, Emond MJ, Loeb LA, Swisher EM, Risques RA. Ultra-deep sequencing detects ovarian cancer cells in peritoneal fluid and reveals somatic TP53 mutations in noncancerous tissues. Proc Natl Acad Sci U S A. 2016;113(21):6005-10.

31. Young AL, Challen GA, Birmann BM, Druley TE. Clonal haematopoiesis harbouring AML-associated mutations is ubiquitous in healthy adults. Nat Commun. 2016;7:12484.

32. Anglesio MS, Papadopoulos N, Ayhan A, Nazeran TM, Noe M, Horlings HM, Lum A, Jones S, Senz J, Seckin T, et al. Cancer-associated mutations in endometriosis without cancer. N Engl J Med. 2017;376(19):1835-48.

33. Xu ZP, Dutra A, Stellrecht CM, Wu C, Piatigorsky J, Saunders GF. Functional and structural characterization of the human gene BHLHB5, encoding a basic helix-loop-helix transcription factor. Genomics. 2002:80(3):311-8.

34. Meller S, Zipfel L, Gevensleben H, Dietrich J, Ellinger J, Majores M, Stein J, Sailer $V$, Jung $M$, Kristiansen $G$, Dietrich D. CDO1 promoter methylation is associated with gene silencing and is a prognostic biomarker for biochemical recurrencefree survival in prostate cancer patients. Epigenetics. 2016;11(12):871-80.

35. Wrangle J, Machida EO, Danilova L, Hulbert A, Franco N, Zhang W, Glockner SC, Tessema M, Van Neste L, Easwaran $\mathrm{H}$, et al. Functional identification of cancer-specific methylation of CDO1, HOXA9, and TAC1 for the diagnosis of lung cancer. Clin Cancer Res. 2014;20(7):1856-64.

36. Shi YX, Wang Y, Li X, Zhang W, Zhou HH, Yin JY, Liu ZQ. Genome-wide DNA methylation profiling reveals novel epigenetic signatures in squamous cell lung cancer. BMC Genomics. 2017;18(1):901.

37. Palles C, Chegwidden L, Li X, Findlay JM, Farnham G, Castro Giner F, Peppelenbosch MP, Kovac M, Adams CL, Prenen H, et al. Polymorphisms near TBX5 and GDF7 are associated with increased risk for Barrett's esophagus. Gastroenterology. 2015;148(2):367-78.

38. Yu J, Ma X, Cheung KF, Li X, Tian L, Wang S, Wu CW, Wu WK, He M, Wang M, et al. Epigenetic inactivation of T-box transcription factor 5, a novel tumor suppressor gene, is associated with colon cancer. Oncogene. 2010;29(49):6464-74.

39. Risinger Jl, Hayes K, Maxwell GL, Carney ME, Dodge RK, Barrett JC, Berchuck A. PTEN mutation in endometrial cancers is associated with favorable clinical and pathologic characteristics. Clin Cancer Res. 1998;4(12):3005-10.

40. Stambolic V, MacPherson D, Sas D, Lin Y, Snow B, Jang Y, Benchimol S, Mak TW. Regulation of PTEN transcription by p53. Mol Cell. 2001;8(2):317-25.

41. Su JD, Mayo LD, Donner DB, Durden DL. PTEN and phosphatidylinositol 3'-kinase inhibitors up-regulate p53 and block tumor-induced angiogenesis: evidence for an effect on the tumor and endothelial compartment. Cancer Res. 2003;63(13):3585-92.

42. Li AG, Piluso LG, Cai X, Wei G, Sellers WR, Liu X. Mechanistic insights into maintenance of high p53 acetylation by PTEN. Mol Cell. 2006;23(4):575-87.

43. Vazquez-Ulloa E, Lizano M, Aviles-Salas A, Alfaro-Moreno E, ContrerasParedes A. Abnormal distribution of hDlg and PTEN in premalignant lesions and invasive cervical cancer. Gynecol Oncol. 2011;122(3):663-8.

44. Salk JJ, Loubet-Senear K, Maritschnegg E, Valentine CC, Williams LN, Higgins JE, Horvat R, Vanderstichele A, Nachmanson D, Baker KT, et al. Ultra-sensitive TP53 sequencing for cancer detection reveals progressive clonal selection in normal tissue over a century of human lifespan. Cell Rep. 2019;28(1):132-44.e3.

\section{Publisher's Note}

Springer Nature remains neutral with regard to jurisdictional claims in published maps and institutional affiliations. 